\title{
SPACES WHICH ADMIT AR-RESOLUTIONS
}

\author{
A. KOYAMA, S. MARDEŠIĆ AND T. WATANABE \\ (Communicated by Doug W. Curtis)
}

\begin{abstract}
It is proved that a topological space $X$ admits an AR-resolution (in the sense of [6]) if and only if $X$ has trivial (strong) shape.
\end{abstract}

1. Introduction. It is well known that every topological space $X$ admits an ANR-resolution in the sense of [6], i.e., a resolution $p=\left(p_{a}\right): X \rightarrow \mathbf{X}=$ $\left(X_{a}, p_{a a^{\prime}}, A\right)$, where each $X_{a}$ is an ANR (for metric spaces) [6, Theorem 12]. A. Koyama raised the question to determine which spaces admit AR-resolutions, i.e., resolutions where each $X_{a}$ is an AR (for metric spaces). Clearly, such spaces must be of trivial shape. In this note we show that the converse also holds. More precisely, we prove the following

THEOREM. Let $X$ be a topological space. Then the following three conditions are equivalent:

(i) $X$ admits an $A R$-resolution.

(ii) $X$ has trivial strong shape.

(iii) $X$ has trivial shape.

Recall that compact metric spaces of trivial shape are limits of inverse sequences of Hilbert cubes $[\mathbf{1}, \mathbf{5}]$.

2. Implication (i) $\Rightarrow$ (ii). We use the notions of coherent map of systems, coherent homotopy and strong shape, ssh, as defined in [2-4].

We first establish a lemma.

LEMMA 1. Let $\mathbf{X}=\left(X_{a}, p_{a a^{\prime}}, A\right)$ and $\mathbf{Y}=\left(Y_{b}, q_{b b^{\prime}}, B\right)$ be inverse systems of metric spaces over (directed) antisymmetric cofinite index sets. If all $Y_{b}$ are AR's, then any two coherent maps $f, f^{\prime}: \mathbf{X} \rightarrow \mathbf{Y}$ are coherently homotopic.

PrOOF. Since $B$ is cofinite, without loss of generality we can assume that $f$ and $f^{\prime}$ are special coherent maps [4, Lemma 5], i.e., are given by increasing functions $\varphi, \varphi^{\prime}: B \rightarrow A$ and by maps $f_{\mathrm{b}}: \Delta^{n} \times X_{\varphi\left(b_{n}\right)} \rightarrow Y_{b_{0}}, f_{\mathrm{b}}^{\prime}: \Delta^{n} \times X_{\varphi^{\prime}\left(b_{n}\right)} \rightarrow Y_{b_{0}}$, respectively; here $\mathbf{b}=\left(b_{0}, \ldots, b_{n}\right), b_{0} \leq \cdots \leq b_{n}$. We choose an increasing function $\Phi: B \rightarrow A$ such that $\Phi \geq \varphi, \varphi^{\prime}$. We need maps $F_{\mathrm{b}}: \Delta^{n} \times I \times X_{\Phi\left(b_{n}\right)} \rightarrow Y_{b_{0}}$ such that $\left(\Phi, F_{\mathbf{b}}\right)$ is a coherent map $F: I \times \mathbf{X} \rightarrow \mathbf{Y}$ satisfying

$$
\begin{aligned}
& F_{\mathbf{b}}(t, 0, x)=f_{\mathbf{b}}\left(t, p_{\varphi\left(b_{n}\right) \Phi\left(b_{n}\right)}(x)\right), \\
& F_{\mathbf{b}}(t, 1, x)=f_{\mathbf{b}}^{\prime}\left(t, p_{\varphi^{\prime}\left(b_{n}\right) \Phi\left(b_{n}\right)}(x)\right) .
\end{aligned}
$$

Received by the editors December 15, 1986.

1980 Mathematics Subject Classification (1985 Revision). Primary 54B25, 54C55, 54C56.

Key words and phrases. Shape, strong shape, resolution, inverse limit, AR-spaces.

This paper is the result of a visit of A. Koyama and T. Watanabe to the University of Zagreb in October 1986 
We construct the maps $F_{\mathrm{b}}=F_{b_{0} \cdots b_{n}}$ by induction on $n$. Since $Y_{b_{0}}$ is an AR, the maps

$$
f_{b_{0}}\left(0 \times p_{\varphi\left(b_{0}\right) \Phi\left(b_{n}\right)}\right): 0 \times X_{\Phi\left(b_{0}\right)} \rightarrow Y_{b_{0}}
$$

and

$$
f_{b_{0}}^{\prime}\left(1 \times p_{\varphi^{\prime}\left(b_{0}\right) \Phi\left(b_{0}\right)}\right): 1 \times X_{\Phi\left(b_{0}\right)} \rightarrow Y_{b_{0}}
$$

extend to a map $F_{b_{0}}: I \times X_{\Phi\left(b_{0}\right)} \rightarrow Y_{b_{0}}$.

Now assume that we have already defined maps $F_{\mathbf{b}}$, where $\mathbf{b}$ has $<n$ terms, $n \geq 1$. We will now define $F_{b_{0} \cdots b_{n}}: \Delta^{n} \times I \times X_{\Phi\left(b_{n}\right)} \rightarrow Y_{b_{0}}$.

We first consider the case when $\left(b_{0}, \ldots, b_{n}\right)$ is nondegenerate, i.e., $b_{j} \neq b_{j+1}$ for all $j$. By the requirement that $F$ be a coherent map (see $[2,4]$ ) and by (1) and (2), the map $F_{b_{0} \cdots b_{n}}$ is already determined on the closed subset $\partial\left(\Delta^{n} \times I\right) \times X_{\Phi\left(b_{n}\right)}$ of $\Delta^{n} \times I \times X_{\Phi\left(b_{n}\right)}$. Since $Y_{b_{0}}$ is an AR, extensions to all of $\Delta^{n} \times I \times X_{\Phi\left(b_{n}\right)}$ exist. We take such an extension for $F_{b_{0} \cdots b_{n}}$.

If $\left(b_{0}, \ldots, b_{n}\right)$ is degenerate, say $b_{j}=b_{j+1}$, then we put

$$
F_{b_{0} \cdots b_{n}}(t, x)=F_{b_{0} \cdots \hat{b}_{j} \cdots b_{n}}\left(\sigma_{j} t, x\right),
$$

where $\left(b_{0}, \ldots, \hat{b}_{j}, \ldots, b_{n}\right)$ is obtained from $\left(b_{0}, \ldots, b_{n}\right)$ by omitting $b_{j}$ and $\sigma_{j}: \Delta^{n} \rightarrow$ $\Delta^{n-1}$ is the $j$ th degeneracy operator.

PROOF OF (i) $\Rightarrow$ (ii). By assumption, $X$ admits an AR-resolution $p=\left(p_{a}\right): X$ $\rightarrow \mathbf{X}=\left(X_{a}, p_{a a^{\prime}}, A\right)$. We can always achieve that $A$ is antisymmetric and cofinite (use the construction described in [7, I, §1.2, Theorem 2]). On the other hand, a single-point space $Y=\{*\}$ also admits an AR-resolution $q=\left(q_{b}\right): Y \rightarrow \mathbf{Y}$ with antisymmetric and cofinite index set, e.g., such is the rudimentary resolution 1: $Y \rightarrow\left(Y_{b_{0}}\right)$, where $Y_{b_{0}}=Y=\{*\}$.

Constant maps $u: X \rightarrow Y$ and $v: Y \rightarrow X$ induce coherent maps $f: \mathbf{X} \rightarrow \mathbf{Y}$ and $g: \mathbf{Y} \rightarrow \mathbf{X}$ (see [4, II, §2]). By Lemma $1, g f \simeq 1$ and $f g \simeq 1$. Therefore, the induced strong shape morphisms $S_{1}(u): X \rightarrow Y$ and $S_{1}(v): Y \rightarrow X$ satisfy

$$
S_{1}(v) S_{1}(u)=1 \quad \text { and } \quad S_{1}(u) S_{1}(v)=1
$$

This shows that the strong shape $\operatorname{ssh}(X)=\operatorname{ssh}(Y)$. Since $Y=\{*\}$, we conclude that $\operatorname{ssh}(X)=0$.

3. Implication (ii) $\Rightarrow$ (iii). This is an immediate consequence of the fact that there exists a functor $S_{2}: \mathrm{ssh} \rightarrow$ sh from the strong shape category ssh to the shape category sh $[4, \mathrm{II}, \S 2]$.

4. Implication (iii) $\Rightarrow$ (i). In the proof we need a simple lemma.

LEMMA 2. Let $p: X \rightarrow Y$ be a mapping from a metric space $X$ into an ANR $Y$. If $p$ is homotopic to a constant map, then there exist an AR $Z$ and a map $r: Z \rightarrow Y$ such that $X \subseteq Z$ is a closed subset and $r \mid X=p$.

PROOF OF LEMMA 2. By the Kuratowski-Woydisławski embedding theorem (see, e.g., [7, I, §3.1, Theorem 2], $X$ can be embedded as a closed subset in a convex set $Z$ of a normed vector space. By the Dugundji extension theorem (see, e.g., [7, I, §3.1, Theorem 3], $Z$ is an AR. Since $p$ is homotopic to a constant map $c: X \rightarrow Y$ and constants extend to all of $Z$, the homotopy extension theorem (see, e.g., [7, I, $\S 3.2$, Theorem 4] proves that also $p: X \rightarrow Y$ extends to a map $r: Z \rightarrow Y$. 
ProOF OF (iii) $\Rightarrow$ (i). Choose an antisymmetric cofinite ANR-resolution $p=$ $\left(p_{a}\right): X \rightarrow \mathbf{X}=\left(X_{a}, p_{a a^{\prime}}, A\right)$ for the space $X$. Since $\operatorname{sh} X=0$, every $a \in A$ admits an $a^{\prime}=\varphi(a) \geq a$ such that

$$
P_{a \varphi(a)} \simeq 0
$$

(the proof of $[7, \mathrm{II}, \S 2.3$, Theorem 7] applies). Since $A$ is cofinite, we can achieve that $\varphi: A \rightarrow A$ is an increasing function.

If $A$ contains an element $a_{0} \in A$ such that $a_{0} \leq a$ implies $a_{0}=a$, then (by directedness and antisymmetry) $a_{0}$ is the unique element $a_{0}=\max A$. In this case $p_{a_{0}}: X \rightarrow\left(X_{a_{0}}\right)$ is also a resolution and since $\varphi\left(a_{0}\right)=a_{0},(1)$ shows that $X_{a_{0}}$ is contractible and therefore an $\mathrm{AR}$, which verifies the assertion.

We will now consider the case where $A$ has no maximal element, i.e., every $a \in A$ admits an $a^{\prime} \geq a$, which is different from $a$.

By Lemma 2 and (1), with each $a \in A$ one can associate an AR $Z_{a}$ and a map $r_{a}: Z_{a} \rightarrow X_{a}$ such that $X_{\varphi(a)} \subseteq Z_{a}$ is a closed subset and

$$
r_{a} \mid X_{\varphi(a)}=p_{a \varphi(a)} \text {. }
$$

We now define a new ordering $\leq^{*}$ in $A$ by putting $a_{1} \leq^{*} a_{2}$ provided $a_{1}=a_{2}$ or $\varphi\left(a_{1}\right) \leq a_{2}$. Clearly, the set $A^{*}=\left(A, \leq^{*}\right)$ is directed, antisymmetric and cofinite. For $a_{1} \leq^{*} a_{2}$ we define a map $q_{a_{1} a_{2}}: Z_{a_{2}} \rightarrow Z_{a_{1}}$ by putting $q_{a_{1} a_{2}}=$ id if $a_{1}=a_{2}$, and

$$
q_{a_{1} a_{2}}=p_{\varphi\left(a_{1}\right) a_{2}} r_{a_{2}} \text { if } a_{1} \leq^{*} a_{2}, \quad a_{1} \neq a_{2} .
$$

Note that in the latter case

$$
q_{a_{1} a_{2}}\left(Z_{a_{2}}\right) \subseteq X_{\varphi\left(a_{1}\right)}
$$

If $a_{1} \leq^{*} a_{2} \leq^{*} a_{3}$, then

$$
q_{a_{1} a_{2}} q_{a_{2} a_{3}}=q_{a_{1} a_{3}} .
$$

This is clear if $a_{1}=a_{2}$ or $a_{2}=a_{3}$. In the remaining case we have

$$
q_{a_{1} a_{2}} q_{a_{2} a_{3}}=p_{\varphi\left(a_{1}\right) a_{2}} r_{a_{2}} p_{\varphi\left(a_{2}\right) a_{3}} r_{a_{3}}=p_{\varphi\left(a_{1}\right) a_{3}} r_{a_{3}}=q_{a_{1} a_{3}},
$$

because (2) implies

$$
r_{a_{2}} p_{\varphi\left(a_{2}\right) a_{3}}=p_{a_{2} \varphi\left(a_{2}\right)} p_{\varphi\left(a_{2}\right) a_{3}}=p_{a_{2} a_{3}} .
$$

We have, thus, proved that $\mathbf{Z}=\left(Z_{a}, q_{a a^{\prime}}, A^{*}\right)$ is an AR-system.

We now define maps $q_{a}: X \rightarrow Z_{a}$ by putting

$$
q_{a}=p_{\varphi(a)}: X \rightarrow X_{\varphi(a)} \subseteq Z_{a} .
$$

Note that $a_{1} \leq^{*} a_{2}$ implies

$$
q_{a_{1} a_{2}} q_{a_{2}}=q_{a_{1}} .
$$

This is obvious if $a_{1}=a_{2}$. In the remaining case, we have, by (2),

$$
q_{a_{1} a_{2}} q_{a_{2}}=p_{\varphi\left(a_{1}\right) a_{2}} r_{a_{2}} p_{\varphi\left(a_{2}\right)}=p_{\varphi\left(a_{1}\right)}=q_{a_{1}} \text {. }
$$

The proof will be completed if we show that $q=\left(q_{a}\right): X \rightarrow \mathbf{Z}$ is a resolution, i.e., has properties (R1) and (R2) (see [6 or 7]). 
ProOF OF (R1). Let $P$ be an ANR, let $\mathcal{U}$ be an open covering of $P$ and $f: X \rightarrow P$ a mapping. By (R1) for $p: X \rightarrow \mathrm{X}$ there exists an $a \in A$ and a map $h: X_{a} \rightarrow P$ such that $h p_{a}$ and $f$ are $U$-near maps, which we denote by

$$
\left(h p_{a}, f\right)<U \text {. }
$$

Consider the map $h^{\prime}=h r_{a}: Z_{a} \rightarrow P$. By (2), we have

$$
h^{\prime} q_{a}=h r_{a} p_{\varphi(a)}=h p_{a \varphi(a)} p_{\varphi(a)}=h p_{a} .
$$

Consequently, (11) becomes the desired relation

$$
\left(h^{\prime} q_{a}, f\right)<U .
$$

ProOF OF (R2). Let $P$ be an ANR and $U$ an open covering of $P$. We choose a covering $U^{\prime}$ of $P$ satisfying (R2) for $p: X \rightarrow \mathbf{X}$. Then $U^{\prime}$ also satisfies (R2) for $q: X \rightarrow \mathbf{Z}$. Indeed, assume that $a \in A^{\prime}=A$ and $h, h^{\prime}: Z_{a} \rightarrow P$ are maps satisfying

$$
\left(h q_{a}, h^{\prime} q_{a}\right)<U^{\prime}
$$

Since $h q_{a}=h p_{\varphi(a)}, h^{\prime} q_{a}=h^{\prime} p_{\varphi(a)}$, we conclude that there is an $a^{\prime} \geq \varphi(a)$ such that

$$
\left(h p_{\varphi(a) a^{\prime}}, h^{\prime} p_{\varphi(a) a^{\prime}}\right)<\mathcal{U} .
$$

Note that $a \leq^{*} a^{\prime}$ so that $q_{a a^{\prime}}$ is defined. By the assumption on $A$, we can choose $a^{\prime} \geq \varphi(a)$ such that $a^{\prime} \neq \varphi(a)$ and therefore, $a^{\prime} \neq a$. It follows that $q_{a a^{\prime}}=p_{\varphi(a) a^{\prime}} r_{a^{\prime}}$ and therefore (15) becomes the desired relation

$$
\left(h q_{a a^{\prime}}, h^{\prime} q_{a a^{\prime}}\right)<\mathcal{U} .
$$

This completes the proof of the Theorem.

\section{REFERENCES}

1. T. A. Chapman, On some applications of infinite-dimensional manifolds to the theory of shape, Fund. Math. 76 (1972), 181-193.

2. Ju. T. Lisica and S. Mardesić, Steenrod-Sitnikov homology for arbitrary spaces, Bull. Amer. Math. Soc. (N.S.) 9 (1983), 207-210.

3. - Coherent prohomotopy and a strong shape category of topological spaces, Proc. Internat. Topology Conf. (Leningrad, 1982), Lecture Notes in Math., vol. 1060, Springer-Verlag, Berlin, 1984, pp. 164-173.

4. __ Coherent prohomotopy and strong shape theory, Glas. Mat. Ser. III 19 (1984), 335-399.

5. S. Mardesic, Decreasing sequences of cubes and compacta of trivial shape, General Topology Appl. 2 (1972), 17-23.

6. _ Approximate polyhedra, resolutions of maps and shape fibrations, Fund. Math. 114 (1981), 53-78.

7. S. Mardesic and J. Segal, Shape theory, North-Holland, Amsterdam, 1982.

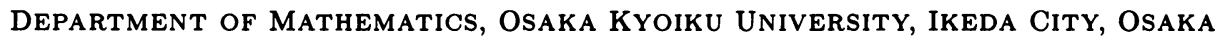
563, JAPAN

Department of MAThematics, UNIVERSity OF ZAGREB, 41001 ZAGREB, P.O.B 187, YUGOSLAVIA

Department of Mathematics, Faculty of Education, University of YamaGUCHI, YAMAGUCHI, JAPAN 